\title{
Prevalencia de infecciones oportunistas en pacientes con VIH-SIDA con base en el estudio microscópico de médula ósea
}

\author{
Prevalence of opportunistic infections in HIV-AIDS \\ patients based on microscopic examination of \\ bone marrow
}

\author{
Roque Julio Corzo, Elsa Marina Rojas, Claudia lucía Figueroa, \\ Nelson Daza • Bucaramanga (Colombia)
}

\section{Resumen}

Introducción: la utilidad diagnóstica del estudio de médula ósea en pacientes con VIH ha sido evaluada en pocos estudios en Latinoamérica. Se desconoce la frecuencia con la que este estudio aporta a la identificación de infecciones oportunistas en nuestro medio.

Diseño: estudio analítico de corte transversal.

Métodos: revisión retrospectiva de historias clínicas de pacientes VIH-SIDA, hospitalizados en el Hospital Universitario de Santander entre 2009 y 2012, quienes fueron sometidos a estudio de médula ósea para evaluar citopenias. Se excluyeron casos con historia de neoplasia y quimioterapia tres meses antes del aspirado. Se calculó tamaño de muestra para evaluación de asociaciones.

Resultados: se evaluaron 92 muestras de médula ósea de 83 pacientes, encontrando una prevalencia de infección oportunista de $23 \%$ con examen directo y $34.5 \%$ cuando se realizaron cultivos. La histoplasmosis fue la infección más frecuente $20.6 \%$, seguida de Mycobacterias $10.8 \%$ e infección por Criptococcus spp 3.2\%. La clínica hematológica que predominó en casos positivos para oportunista fue pancitopenia febril en $41 \%$ y monocitopenia en $32 \%$. La deshidrogenasa láctica $\geq 600 \mathrm{UI} / \mathrm{dL}$, se asoció con un diagnóstico positivo para infección oportunista en el estudio microscópico de médula ósea, con OR 5.42 (IC95\% 1.09-26.9 p =0.03).

Conclusión: el estudio de médula ósea en los pacientes con VIH-SIDA permitió un diagnóstico de infección oportunista en una cuarta parte de los casos, siendo especialmente útil para la identificación temprana de histoplasmosis. Se requieren estudios para evaluar el impacto en estancia hospitalaria, desenlaces clínicos y costos del uso de este examen de forma temprana en el abordaje de pacientes VIH hospitalizados en medicina interna. (Acta Med Colomb 2015; 40: 93-100).

Palabras clave: síndrome de inmunodeficiencia adquirida, examen microscópico de médula ósea, infección oportunista, utilidad diagnóstica.

\footnotetext{
Abstract

Introduction: the diagnostic utility of the study of bone marrow in patients with HIV has been evaluated in a few studies in Latin America. The frequency with which this study contributes to the identification of opportunists in our country is unknown.

Design: Analytical cross-sectional study.

Methods: a retrospective review of medical records of HIV-AIDS patients hospitalized at the University Hospital of Santander between 2009 and 2012 who underwent bone marrow study to evaluate cytopenia. Cases with a history of malignancy and chemotherapy three months before vacuuming were excluded. Sample size for evaluation of partnerships was calculated.

Results: 92 samples of bone marrow of 83 patients were evaluated, finding a prevalence of opportunistic infection in $23 \%$ with direct examination and $34.5 \%$ when cultures were performed. Histoplasmosis was the most common infection with $20.6 \%$, followed by $10.8 \%$ Mycobacteria and Cryptococcus spp infection $3.2 \%$. The hematologic clinic that prevailed in the positive cases for
}

Dr. Roque Julio Corzo Corzo: Especialista en Medicina Interna. Universidad Industrial de Santander-UIS; Dra. Elsa Marina Rojas Garrido: Especialista en Medicina Interna UIS. Infectóloga Universidad de Antioquia. Coordinadora de Posgrado de Medicina Interna UIS; Dra. Claudia Lucía Figueroa Pineda: Especialista en Medicina Interna UIS. Magister en Epidemiología UIS; Dr Nelson Daza: Internista-Hematólogo. Director Banco Metropolitano de Sangre. Hospital Universitario de Santander. Bucaramanga (Colombia).

Correspondencia. Dr Roque Julio Corzo Corzo. Bucaramanga (Colombia).

E-mail: roquecorzo@yahoo.com

Recibido: 21/VIII/2014 Aceptado: 23/II/2015 
opportunist was pancitopenia febril (in $41 \%$ of the cases) and monocitopenia (in $32 \%$ of the cases). The $\mathrm{LDH} \geq 600 \mathrm{IU} / \mathrm{dL}$ was associated with a positive diagnosis for opportunistic infection in the microscopic study of bone marrow, with OR $5.42(95 \%$ CI $1.09-26.9 \mathrm{p}=0.03)$.

Conclusion: the study of bone marrow in patients with HIV-AIDS allowed a diagnosis of opportunistic infection in a quarter of cases, and is especially useful for early identification of histoplasmosis. Studies to assess the impact on hospital stay, clinical outcomes and costs of using this test early in the approach to HIV patients hospitalized in Internal Medicine are required. (Acta Med Colomb 2015; 40: 93-100).

Keywords: acquired immunodeficiency syndrome, microscopic examination of bone marrow, opportunistic infection, diagnostic utility.

\section{Introducción}

El conocimiento del aporte de las pruebas diagnósticas que permiten realizar un diagnóstico acertado y temprano de las entidades oportunistas que afectan a los pacientes con infección por el virus de la inmunodeficiencia humana (VIH) es fundamental en el abordaje de esta población. En la literatura se encuentran algunos estudios, que han evaluado la utilidad diagnóstica del estudio de médula ósea (1-3). La mayoría de éstos se han realizado con la indicación de síndrome febril de origen desconocido y algunos para evaluación de citopenias (4-6). De acuerdo con estos estudios el aporte al diagnóstico varía según diferentes áreas geográficas, así como el comportamiento epidemiológico de ciertas condiciones como tuberculosis e histoplasmosis. Igualmente se ha tratado de evaluar variables clínicas y paraclínicas como potenciales factores de predicción de un resultado positivo para infección oportunista en médula ósea, siendo los parámetros hematológicos y las pruebas de función hepática los que más se han relacionado con este desenlace mas no de forma consistente en todos los estudios $(2,7,8)$.

El Hospital Universitario de Santander (HUS) atiende el mayor número de pacientes con la condición VIH-SIDA en el oriente del país. En revisiones preliminares no se encontraron registros de este centro hospitalario, ni otros en la región o Colombia en relación con la evaluación microscópica de médula ósea en pacientes con VIH-SIDA. Por esta razón el objetivo de este estudio fue determinar la prevalencia de infecciones oportunistas con base en el estudio microscópico de médula ósea y así mismo caracterizar con aspectos clínicos y pruebas diagnósticas complementarias a los pacientes sometidos a dicho estudio.

\section{Material y métodos}

Estudio epidemiológico descriptivo, retrospectivo, analítico de corte transversal. La población correspondió a pacientes con diagnóstico de VIH-SIDA que fueron institucionalizados en el servicio de medicina interna del HUS entre enero de 2009 y diciembre de 2012 y llevados a estudio de médula ósea.

El tamaño de muestra se determinó con el promedio de prevalencias de infecciones oportunistas $(18.1 \%)$ reportadas en estudios similares desarrollados en Latinoamérica (4, 6, 9). Se estimó una muestra total de 92 pacientes y/o estudios de médula ósea. Con el fin de evaluar la posible asociación entre la variable de interés lactato deshidrogenasa (LDH) y la positividad para infección oportunista, se estimó un segundo tamaño de muestra de acuerdo con la prevalencias reportadas para histoplasmosis, toxoplasmosis y pneumocistosis (10-12). Por el método de Fleis se determinó evaluar 53 pacientes, 11 expuestos a la variable de interés ( $\mathrm{LDH}>600 \mathrm{UI} / \mathrm{dL}$ ) y 42 no expuestos $(\mathrm{LDH}<600 \mathrm{UI} / \mathrm{dL})$. Los pacientes se identificaron de los registros del servicio de hematología del HUS entre 2009 y 2012, con los cuales se elaboró una base de datos que quedó conformada por información obtenida de las historias clínicas que fueron suministradas por el servicio de estadística del hospital y que cumplieron los criterios de inclusión.

Se incluyó todo paciente hospitalizado mayor de 13 años con prueba Western Blot positiva para VIH, que fueron sometidos a estudio microscópico de médula ósea, sin importar la indicación. El estudio microscópico de médula ósea incluyó la realización de aspirado, biopsia y/o ambas condiciones. Se incluyeron los pacientes con una de las siguientes tres condiciones: 1) pacientes con aspirado más biopsia de médula ósea, 2) sólo aspirado, 3) sólo biopsia. La realización de mielocultivos no se consideró un criterio de inclusión. Se excluyeron los pacientes con patología neoplásica que hubieran recibido tratamiento con quimioterapia en los tres meses previos a la hospitalización y aquellos cuyo estudio de aspirado y/o biopsia, no fueron aptos para lectura por el hematólogo y/o patólogo.

Se revisó información de forma retrospectiva procedente de las historias clínicas donde se constataron los datos demográficos, clínicos y resultados de estudios paraclínicos de acuerdo con el formato de recolección de datos diseñado. Se midieron variables clínicas y paraclínicas presentes al ingreso y/o hospitalización en la cual se realizó el estudio de médula ósea. Se evaluó la presencia de hepatoesplenomegalia, hepatomegalia y esplenomegalia, determinado por el examen físico y estudio de imágenes diagnósticas de ingreso o durante la hospitalización. Se tomaron datos de paraclínicos realizados a su ingreso o los datos disponibles en el sistema de información del hospital, siempre y cuando 
antecedieran por fecha a la realización del estudio de médula ósea. Se midieron las siguientes variables: hemoglobina, recuento leucocitario, neutropenia, recuento plaquetario, creatinina, proteína $\mathrm{C}$ reactiva, lactato deshidrogenasa (LDH), aspartato transaminasa (AST) y alanino transaminasa (ALT), bilirrubina total, bilirrubina directa, fosfatasa alcalina, ferritina y albúmina.

Con respecto al estudio microscópico de médula ósea se describieron las principales alteraciones reportadas en el aspirado, biopsia y/o ambos. De la impronta de la biopsia se reportó como normal, muestra insuficiente y/o alterada y se describieron los hallazgos. Se evaluó la positividad de las tinciones en la biopsia de médula ósea para hongos (ácido periódico Schiff (PAS), plata metenamina) y ZiehlNeelsen. En los casos que se realizaron mielocultivos, éstos se reportaron como negativos o positivos.

Con el objeto de definir un diagnóstico positivo para infección oportunista en el estudio microscópico y microbiológico de médula ósea, se estableció el cumplimiento de definiciones operativas.

\section{Análisis estadístico}

Los datos fueron recolectados por un solo investigador e ingresados a una base de datos en una hoja de cálculo Excel 2007 y analizados usando el programa estadístico Stata11.1. Las variables cuantitativas se expresaron en medidas de tendencia central y medidas de dispersión. Las variables categóricas fueron analizadas utilizando Chi Square o Test de Fisher y las variables continuas fueron analizadas usando T-student. Para comparar variables no paramétricas se utilizó el test de Mann-Whitney y para análisis de asociación regresión logística. Se realizaron pruebas de significancia estadística asumiendo como significativo un valor $\mathrm{p}<0.05$. Con el fin de evaluar posible asociación entre variables clínicas y paraclínicas con el desenlace de estudio positivo para infección oportunista, se calcularon OR (Odds Ratio) e intervalos de confianza del $95 \%$.

El presente estudio fue aprobado por el comité de ética en investigación científica de la Universidad Industrial de Santander y fue clasificado como investigación "sin riesgo".

\section{Resultados}

Se revisaron las historias clínicas de 83 pacientes que aportaron un total de 92 estudios de médula ósea durante un periodo de cuatro años. El $72 \%$ eran hombres y $28 \%$ mujeres. El promedio de edad para la población fue 38.7 años (DE, 10.9 años). El 69.5\% de los pacientes procedían del área metropolitana de Bucaramanga y $23.9 \%$ de otros municipios de Santander (Tabla 1).

El promedio de hemoglobina fue $8.88 \mathrm{~g} / \mathrm{dL}$ (DE, 2.23). La mediana del recuento de leucocitos fue $3400 / \mathrm{mm}^{3}$ con RIC 25\%-75\% (2000-5200). Un valor mínimo de 700 y máximo de 27800 . Cerca de $60 \%$ tenían un recuento menor a 4000 leucocitos $/ \mathrm{cm}^{3}$. En $30.43 \%$ de los estudios de médula ósea se encontró neutropenia y $30.2 \%$ con trombo-
Tabla 1. Características sociodemográficas y clínicas de los pacientes con VIH-SIDA sometidos a estudio de médula ósea.

\begin{tabular}{|c|c|}
\hline Características & n $(\%)$ \\
\hline \multicolumn{2}{|l|}{ Edad-años } \\
\hline Media (DE) & $38.7(10.9)$ \\
\hline \multicolumn{2}{|l|}{ Sexo } \\
\hline Hombres & $60(72)$ \\
\hline Mujeres & $23(28)$ \\
\hline \multicolumn{2}{|l|}{ Estado civil } \\
\hline Soltero & $35(38)$ \\
\hline Separado & $14(15)$ \\
\hline Unión libre & $11(12)$ \\
\hline Sin datos & $32(34.7)$ \\
\hline \multicolumn{2}{|l|}{ Lugar de procedencia } \\
\hline Bucaramanga y área metropolitana & $64(69.5)$ \\
\hline Otro municipio de Santander & $22(24)$ \\
\hline Otro departamento & $6(6.5)$ \\
\hline \multicolumn{2}{|l|}{ Historia médica previa } \\
\hline \multicolumn{2}{|l|}{ Diagnóstico de infección por VIH } \\
\hline Diagnóstico previo & $69(75)$ \\
\hline Diagnóstico de Novo & $23(25)$ \\
\hline Datos de carga viral en los últimos seis meses & $27(29.5)$ \\
\hline Recuento de CD4 + en los últimos seis meses & $30(32.5)$ \\
\hline Tratamiento antirretroviral actual & $37(40)$ \\
\hline Tratamiento con zidovudina (AZT) & $17(18.4)$ \\
\hline Terapia profiláctica con trimetoprim-sulfametoxazol & $37(40)$ \\
\hline Infección oportunista en médula ósea previa & $5(5.4)$ \\
\hline
\end{tabular}

citopenia. La media del recuento plaquetario fue 184576.7 (DE, 133 287.3). Las demás características de laboratorio están representadas en la Tabla 2.

Cuatro pacientes tenían prueba positiva para el antígeno de superficie. En cuatro pacientes se encontró VDRL reactiva, los cuales fueron clasificados como sífilis en fase indeterminada.

En los estudios de medula ósea aportados por los 83 pacientes, se encontró compromiso hepatoesplénico en $41(44.5 \%)$. En $30(32.61 \%)$ se documentó hepatoesplenomegalia a su ingreso en la hospitalización en la cual se realizó el estudio de médula ósea.

Se evaluaron 92 estudios de médula ósea correspondientes a 83 pacientes con diagnóstico de VIH-SIDA. De estos pacientes, siete tenían como mínimo dos estudios realizados en hospitalizaciones diferentes, seis con dos estudios de médula ósea y un caso con cuatro estudios realizados en diferentes hospitalizaciones.

De acuerdo con los registros realizados se encontró que en $61(66 \%)$ se practicó aspirado, biopsia y algún tipo de mielocultivo. En 25 (27\%) se realizó aspirado más biopsia sin mielocultivos (Figura 1).

A todos los pacientes se les realizó aspirado. En 15 (16.3\%) la muestra del aspirado fue reportada como diluida. En 12 casos (13\%) no se encontró información del resultado 
Tabla 2. Características de laboratorio de los pacientes con VIH-SIDA sometidos a estudio de médula ósea.

\begin{tabular}{|c|c|c|c|c|c|}
\hline \multirow[t]{2}{*}{ Variable } & \multirow[t]{2}{*}{ \% Población } & \multirow[t]{2}{*}{ Promedio (DE) } & \multirow[t]{2}{*}{ Mediana } & \multicolumn{2}{|c|}{ RIQ } \\
\hline & & & & 25 & 75 \\
\hline Hemoglobina g/dL & 100 & $8.8(2.23)$ & 9.02 & 7.6 & 9.92 \\
\hline Leucocitos $/ \mathrm{mm}^{3}$ & 100 & $4099.6(3519.3)$ & 3400 & 2000 & 5200 \\
\hline Plaquetas $/ \mathrm{mm}^{3}$ & 93 & 184576.7 (133 287.3) & 154500 & 83000 & 253000 \\
\hline Neutrófilos $/ \mathrm{mm}^{3}$ & 98.9 & $3152.9(2912.2)$ & 2449 & 1340 & 3910 \\
\hline Creatinina $\mathrm{mg} / \mathrm{dL}$ & 91 & $1.14(1.08)$ & 0.92 & 0.74 & 1.12 \\
\hline Proteína $\mathrm{C}$ reactiva $\mathrm{mg} / \mathrm{L}$ & 54 & $112.5(105)$ & 96 & 24 & 192 \\
\hline LDH UI/dL & 93 & $1144.5(1981.4)$ & 618 & 360 & 1263 \\
\hline ASAT UI/L & 88 & $101.3(127)$ & 61.6 & 32.3 & 131.5 \\
\hline ALAT UI/L & 88 & $60.6(109.2)$ & 35.2 & 22.75 & 65.1 \\
\hline Bilirrubina total $\mathrm{mg} / \mathrm{dL}$ & 65 & $0.98(1.57)$ & 0.52 & 0.34 & 0.84 \\
\hline Bilirrubina directa $\mathrm{mg} / \mathrm{dL}$ & $\begin{array}{l}6 \\
3\end{array}$ & $0.62(1.19)$ & 0.23 & 0.19 & 0.43 \\
\hline Fosfatasa alcalina UI/L & 51 & $630.7(1106.2)$ & 325 & 205 & 752 \\
\hline Albumina $\mathrm{g} / \mathrm{dL}$ & 55 & $2.52(0.73)$ & 2.4 & 1.96 & 3.02 \\
\hline
\end{tabular}

del estudio. Finalmente se evaluaron los resultados de 65 estudios de aspirados médula ósea (70.65\%) que estuvieron disponibles en los archivos de la unidad de hematología y en las historias clínicas de los pacientes. De éstos, en 10 casos la lectura fue realizada en la impronta de médula ósea, en 44 casos (67.6\%) la lectura se realizó con base en el aspirado y la impronta y en los casos restantes (11), la lectura fue basada sólo en el aspirado.

En 51 aspirados de médula ósea, 78.4\% presentaron alguna alteración hematológica. Las alteraciones morfológicas generales más frecuentes fueron: plasmocitosis reactiva (78\%), diseritropoyesis $(30.4 \%)$, hiperplasia de la línea eritroide $(23 \%)$ y megaloblastosis $(6.1 \%)$. En 10 aspirados de

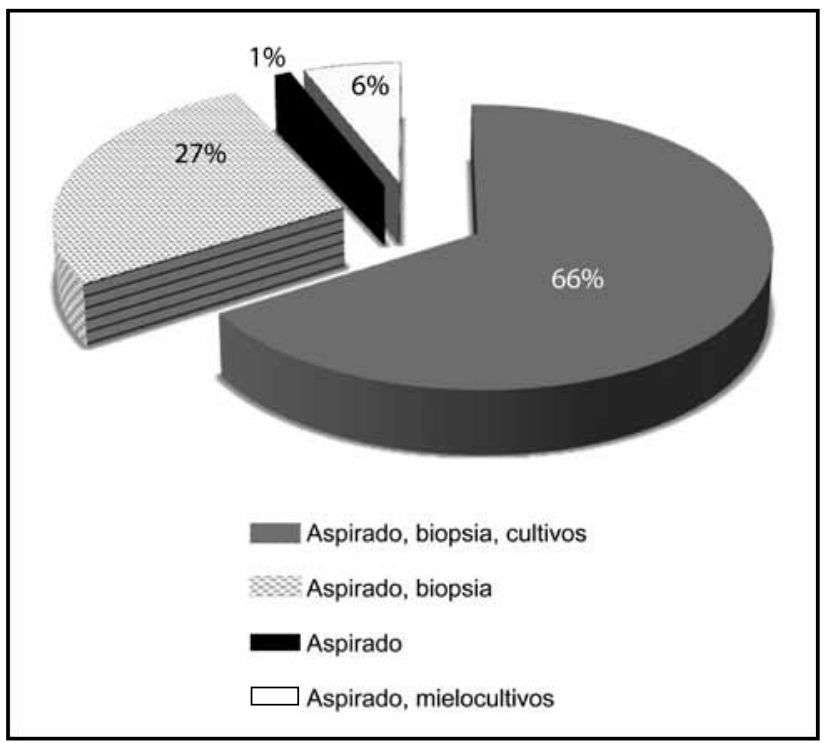

Figura 1. Estudio microscópico y microbiológico de médula ósea. médula ósea correspondientes a $15.3 \%$, se encontró hallazgos que sugirieron el diagnóstico de infección oportunista en médula ósea de acuerdo con las definiciones operativas realizadas. En $100 \%$ de éstos, se encontró compromiso por Histoplasma capsulatum.

En relación con el estudio histopatológico de médula ósea, se evaluaron 86 biopsias que fueron realizadas en los 83 pacientes que ingresaron al estudio. De éstas, cuatro casos presentaron muestra insuficiente para interpretación. En 79 biopsias $(91.8 \%)$, se determinó la celularidad, encontrándose médula ósea normal en $40.5 \%$, hipocelular en $49.3 \%$, hipercelular en $8.8 \%$ y un caso con aplasia celular. De las 82 biopsias disponibles y aptas para interpretación, el estudio histopatológico se evidenció infección oportunista en 19 casos $(23.1 \%)$. Se encontraron formas micóticas compatibles con Histoplasma capsulatum en 15 de éstos (18.2\%). Los cuatro casos restantes, en tres se evidenció inflamación granulomatosa que sugirió compromiso por micobacterias, y un caso en el que se visualizó estructuras micóticas compatibles con criptococo. La presencia de granulomas fue informada en cuatro estudios de biopsia (4.8\%). De éstos, dos casos presentaban necrosis caseificante central. El hallazgo de granuloma fue asociado en tres casos con infección por micobacterias y un caso con Histoplasma capsulatum.

Los 92 estudios de médula ósea se categorizaron de acuerdo con la condición de citopenias. Ésta a su vez fue definida de acuerdo con los parámetros del hemograma de ingreso. De esta manera se encontró que 34 (36.95\%) de los estudios fueron realizados bajo la condición de anemia y leucopenia, $21(22.8 \%)$ pancitopenia y 30 (32.6\%) con anemia aislada. Así mismo se clasificaron los estudios de médula ósea de acuerdo con la condición de citopenias y fiebre, dividiéndolos en cuatro categorías las cuales están descritas en la Tabla 3. 
Tabla 3. Categorización de los estudios de médula ósea de acuerdo con la condición de citopenias y fiebre.

\begin{tabular}{|l|c|c|}
\hline Indicación & $\mathbf{n}(\boldsymbol{\%})$ & $\begin{array}{c}\text { Casos positivos en el estudio } \\
\text { microscópico de médula ósea } \\
\mathbf{n = 2 2} \mathbf{n}(\%)\end{array}$ \\
\hline Fiebre origen desconocido & $1(1.09)$ & - \\
\hline Pancitopenia febril & $19(20.65)$ & $9(40.9)$ \\
\hline Pancitopenia afebril & $2(2.17)$ & - \\
\hline Bicitopenia febril & $26(28.2)$ & $5(22.7)$ \\
\hline Bicitopenia afebril & $12(13)$ & $1(4.5)$ \\
\hline Monocitopenia & $32(34.78)$ & $7(31.8)$ \\
\hline
\end{tabular}

La prevalencia de infección oportunista con base en el estudio microscópico de médula ósea (aspirado y/o biopsia) fue de 23.9\% (IC 95\%:15-32.7). Ésto representa 22 casos positivos. De éstos, en $18(81.8 \%)$ se visualizaron estructuras compatibles con Histoplasma capsulatum, en tres casos se encontró inflamación granulomatosa crónica que sugirió compromiso por micobacterias y en un caso se observó estructuras micóticas compatibles con criptococo. En 17 casos (77.2\%) el estudio microscópico de médula ósea fue la prueba que realizó el diagnóstico de estas condiciones.

Al tener en cuenta el estudio microscópico de médula ósea (aspirado y/o biopsia) y los mielocultivos que fueron disponibles, se encontraron 29 casos positivos para infección oportunista. De éstos, se documentó más de una infección oportunista en tres casos, por lo cual la cifra de casos positivos asciende a 32, lo que corresponde a una prevalencia de infección oportunista de $34.5 \%$ con base en el estudio microscópico y microbiológico de médula ósea. Los 32 casos de infecciones oportunistas corresponden a 16 casos positivos para histoplasmosis como germen único, ocho casos para compromiso diseminado por micobacterias, dos casos positivos para criptococosis diseminada como germen único, y en tres pacientes se encontró estudio positivo para dos entidades oportunistas (dos de éstos corresponden a la documentación simultánea de histoplasmosis y micobacterias no tuberculosas y el restante caso positivo para histoplasmosis y criptococo) (Tablas 4 y 5). Dentro de los pacientes con dos o más muestras, la prevalencia de infección oportunista con base en el estudio microscópico de médula ósea fue $37.5 \%$.
En relación con el estudio microbiológico de médula ósea, en 63 estudios se realizó cultivos para gérmenes comunes $(68.4 \%)$. De éstos, cinco fueron positivos y cinco informados como contaminados. De los estudios positivos en tres se aisló Criptococcus spp, un aislamiento de Histoplasma capsulatum y en otro se aisló Aeromona sobria. Los cultivos para identificación de micobacterias fueron realizados en 56 estudios (60.8\%). Se encontraron nueve estudios positivos para micobacterias, un estudio positivo para criptococo, un aislamiento de Aeromona sobria y cuatro estudios informados como contaminados. De los aislamiento de micobacterias se encontraron cuatro estudios con $\mathrm{Myco}$ bacterium spp, un aislamiento de Mycobacterium avium intracelular, tres estudios con Mycobacterium tuberculoso y uno con Mycobacterium abscessus. En 52 muestras (56.5\%), se realizaron cultivos para hongos en médula ósea. De éstos, tres estudios fueron positivos para Histoplasma capsulatum, tres contaminados y un estudio con aislamiento de Aeromona sobria. Los cultivos positivos para Aeromona sobria corresponden a un solo paciente, cuyo hallazgo microbiológico fue considerado como contaminación.

De acuerdo con la condición de citopenias y la presencia de fiebre se presentó mayor porcentaje de casos positivos en el estudio microscópico de médula ósea para infección oportunista en aquellos pacientes con la condición de pancitopenia febril y monocitopenia (Tabla 3). Así mismo los gérmenes documentados en el estudio microscópico de médula ósea (aspirado más biopsia) de acuerdo con la categorización de citopenias y fiebre se describen en la Tabla 6 .

Tabla 5. Total de casos positivos para infección oportunista en el estudio microscópico y microbiológico de médula ósea y tipo de germen identificado.

\begin{tabular}{|c|c|}
\hline Germen oportunista & $\mathbf{n}$ \\
\hline \multicolumn{2}{|l|}{ Casos positivos con germen único } \\
\hline Histoplasma capsulatum & 16 \\
\hline Mycobacterium spp & 4 \\
\hline Mycobacterium tuberculoso & 3 \\
\hline Ziehl-Neelsen (+) en biopsia con mielocultivo (-) & 1 \\
\hline Cryptococus spp & 1 \\
\hline Cryptococus neoformans & 1 \\
\hline \multicolumn{2}{|l|}{ Casos positivos con dos gérmenes } \\
\hline Histoplasma capsulatum mas Mycobacterium abscessus & 1 \\
\hline Histoplasma capsulatum, más Mycobacterium avium intracelular & 1 \\
\hline Histoplasma capsulatum, más Cryptococus neoformans & 1 \\
\hline
\end{tabular}

Tabla 4. Casos positivos de infecciones oportunistas de acuerdo a la categorización del estudio de médula ósea.

\begin{tabular}{|c|c|c|c|c|c|c|c|c|}
\hline \multirow[t]{2}{*}{ Germen oportunista } & \multirow[t]{2}{*}{$\mathbf{n}$} & \multicolumn{7}{|c|}{ Estudio microscópico y microbiológico de médula ósea } \\
\hline & & Aspirado & Biopsia & $\underset{\text { biopsia }}{\text { Aspirado + }}$ & $\begin{array}{l}\text { Aspirado + } \\
\text { cultivo }\end{array}$ & $\begin{array}{c}\text { Aspirado + } \\
\text { biopsia + cultivo }\end{array}$ & $\begin{array}{c}\text { Biopsia + } \\
\text { cultivo }\end{array}$ & Mielocultivo \\
\hline Histoplasma capsulatum & 19 & 3 & 7 & 6 & 1 & 1 & -- & 1 \\
\hline Micobacterium spp & 10 & -- & 1 & -- & -- & -- & 2 & 7 \\
\hline Criptococcus spp & 3 & -- & 1 & -- & -- & -- & -- & 2 \\
\hline Total & 32 & 3 & 9 & 6 & 1 & 1 & 2 & 10 \\
\hline
\end{tabular}


Tabla 6. Gérmenes oportunistas documentados en el estudio microscópico de médula ósea (aspirado y/o biopsia) de acuerdo con la condición de citopenias y fiebre.

\begin{tabular}{|c|c|c|}
\hline Citopenias & $\begin{array}{c}\text { Casos positivos } \\
\text { n }(\%)\end{array}$ & $\begin{array}{c}\text { Germen oportunista } \\
\text { n }(\%)\end{array}$ \\
\hline \multicolumn{3}{|l|}{ Pancitopenia } \\
\hline Pancitopenia febril & $9(41)$ & $\begin{array}{l}\text { Histoplasma capsulatum (8) } \\
\text { Criptococcus (1) }\end{array}$ \\
\hline \multicolumn{3}{|l|}{ Bicitopenia febril } \\
\hline Anemia leucopenia & \multirow[t]{3}{*}{$5(22.7)$} & Histoplasma capsulatum (2) \\
\hline Anemia trombocitopenia & & Histoplasma capsulatum (2) \\
\hline Leucopenia & & Histoplasma capsulatum (1) \\
\hline \multicolumn{3}{|l|}{ Bicitopenia afebril } \\
\hline Anemia leucopenia & $1(4.6)$ & Histoplasma capsulatum (1) \\
\hline \multicolumn{3}{|l|}{ Una línea celular } \\
\hline Anemia & \multirow[t]{2}{*}{$7(31.8)$} & $\begin{array}{l}\text { Histoplasma capsulatum (4) } \\
\text { Mycobacterium tuberculosis (2) }\end{array}$ \\
\hline Leucopenia & & Histoplasma capsulatum (1) \\
\hline
\end{tabular}

En siete casos se documentaron enfermedades hematooncológicas en la hospitalización en la que se realizó el estudio. De éstos, tres casos correspondieron a linfoma no Hodking, dos casos a linfoma Hodking y dos casos de leucemia aguda.

De los 92 estudios de médula ósea, en 86 (92\%) de éstos se encontró datos de la variable lactato deshidrogenasa, 42 tenían un nivel de LDH por debajo de $600 \mathrm{UI} / \mathrm{dL}$ y 44 presentaban niveles de LDH igual o superior. Con base en estos datos, se incluyó la totalidad de los estudios de médula ósea con LDH por debajo de $600 \mathrm{UI} / \mathrm{dL}$ (42) y se realizó aleatorización simple en los 44 estudios de médula ósea con
Tabla 8. Análisis bivariado expresado en OR entre los casos positivos y negativos para infección oportunista de acuerdo al estudio microscópico de médula ósea.

\begin{tabular}{|l|c|c|c|c|c|}
\hline Variable & $\mathbf{n}$ & $\%$ & OR & IC95\% & P \\
\hline Leucocitos $\leq 1500 / \mathrm{mm}^{3}$ & 92 & 100 & 3.87 & $(1.26-11.8)$ & 0.01 \\
\hline Neutrófilos $<1500 / \mathrm{mm}^{3}$ & 92 & 100 & 3.11 & $(1.14-8.4)$ & 0.02 \\
\hline AST $\geq 100 \mathrm{UI} / \mathrm{dL}$ & 81 & 88 & 7.15 & $(2.3-21.9)$ & 0.001 \\
\hline ALT $\geq 100 \mathrm{UI} / \mathrm{dL}$ & 80 & 87 & 6.64 & $(2.14-20.5)$ & 0.001 \\
\hline
\end{tabular}

la variable de exposición, es decir, LDH igual o superior a $600 \mathrm{UI} / \mathrm{dL}$, para obtener los 11 estudios de médula ósea requeridos para el análisis.

Se encontró que tener LDH mayor o superior a $600 \mathrm{UI} / \mathrm{dL}$, se asoció cinco veces más con un resultado positivo para infección oportunista (OR de 5.42 IC95\% 1.09-26.9 p =0.03); con similar tendencia a la calculada a través de RR: 3.81 .

Al comparar la totalidad de los casos positivos y negativos para infección oportunista de acuerdo con el estudio microscópico de médula ósea, se encontraron diferencias estadísticamente significativas en el recuento de leucocitos, la presencia de neutropenia, niveles de AST y ALT (Tabla 7). Se estimaron medidas de asociación entre las variables estadísticamente significativas calculando OR (Tabla 8).

\section{Discusión}

Se realizó un estudio descriptivo analítico retrospectivo que evaluó la prevalencia de infecciones oportunistas con base en el estudio de médula ósea. Así mismo se exploró la posible relación de la variable LDH y el desenlace de

Tabla 7. Análisis bivariado entre los casos positivos y negativos para infección oportunista de acuerdo al estudio microscópico de médula ósea.

\begin{tabular}{|c|c|c|c|c|}
\hline \multirow[t]{2}{*}{ Variable } & \multirow[t]{2}{*}{$\mathbf{n}=\mathbf{9 2}$} & \multicolumn{2}{|c|}{ Resultado para oportunismo en estudio microscópico de médula ósea } & \multirow[t]{2}{*}{ Valor de } \\
\hline & & $\begin{array}{c}\text { Positivo } \\
\% \text { o DE o m o p }\end{array}$ & $\begin{array}{ll} & \text { Negativo } \\
\% & \text { o DE o m o p }\end{array}$ & \\
\hline Edad-años (DE) & 92 & $39.6(11.1)$ & $38.4(10.8)$ & 0.6407 \\
\hline Sexo $(\%)$ & 92 & $\begin{array}{l}\mathrm{H}: 72.7 \\
\mathrm{M}: 27.2\end{array}$ & $\begin{array}{l}\mathrm{H}: 71.43 \\
\mathrm{M}: 28.5\end{array}$ & 0.9 \\
\hline $\begin{array}{l}\text { Lugar de residencia } \\
\text { Bucaramanga y área metropolitana }(\%)\end{array}$ & 92 & 68.18 & 70 & 0.87 \\
\hline Diagnóstico VIH de novo (\%) & 92 & 22.73 & 25.7 & 0.77 \\
\hline Antecedente Oportunismo de médula ósea (\%) & 92 & 9.09 & 4.29 & 0.38 \\
\hline Terapia antirretroviral (\%) & 92 & 36.36 & 41.43 & 0.67 \\
\hline Hepatoesplenomegalia (\%) & 92 & 45.45 & 28.57 & 0.141 \\
\hline Hemoglobina g/dL (DE) & 92 & $8.83(2.47)$ & $8.89(2.17)$ & 0.9 \\
\hline Recuento leucocitos/mm³ (RIQ 25-RIQ 75) & 92 & $2300(1300-4100)$ & $3650(2200-5300)$ & 0.04 \\
\hline Neutropenia (\%) & 92 & 50 & 24.29 & 0.022 \\
\hline Recuento de plaquetas $/ \mathrm{mm}^{3}$ (DE) & 86 & $146550(120839.9)$ & $197648.4(135732.6)$ & 0.06 \\
\hline LDH (UI/dL) (RIQ 25-RIQ75) & 86 & $1364(618-2397)$ & $539(330-787)$ & 0.0010 \\
\hline AST(UI/dL) (RIQ25-RIQ75) & 81 & $139.3(75.6-183.3)$ & $51.1(29-99)$ & 0.0003 \\
\hline ALT UI/dL (RIQ25-RIQ75) & 80 & $43.6(38-72)$ & $33(19-59.1)$ & 0.0295 \\
\hline
\end{tabular}


tener un estudio positivo para infección oportunista en médula ósea.

El estudio microscópico de médula ósea (aspirado y biopsia) es una de las pruebas que hacen parte del arsenal de métodos diagnósticos de la fiebre de origen desconocido y citopenias en los pacientes con VIH-SIDA (13-14). La utilidad diagnóstica del estudio de médula ósea (aspirado, biopsia y cultivos) ha oscilado entre 19 y $47 \%(2,4,7,13-$ 17). En el presente estudio hemos documentado que durante el curso de cuatro años en el HUS, el estudio microscópico de médula ósea permitió el diagnóstico de entidades infecciosas y no infecciosas en 24 casos (26.08\%), 22 de estos casos fueron positivos para infección oportunista, lo que denota una prevalencia de infección de $23.9 \%$ (IC 95\% 15-32.7). Ésta a su vez asciende a 32 casos de infección oportunista, lo que corresponde a una prevalencia de $34.5 \%$, si se tiene en cuenta los resultados de los estudios microbiológicos. En concordancia con la literatura esta prevalencia se encuentra en el rango de lo reportado en Latinoamérica $(4,6,9)$.

Sin embargo, difiere de lo reportado por Morais J y cols. (9) quienes realizaron un estudio comparativo en Brasil y evaluaron el impacto de la introducción de la terapia antirretroviral en el aporte diagnóstico del estudio de médula ósea entre dos periodos, y encontraron que la prevalencia de infección oportunista en el estudio de médula ósea disminuyó significativamente de 16-2\% en el segundo periodo (9). De otro lado, en un estudio prospectivo realizado en México por Madrigal H y cols. (6) se reportó una utilidad diagnóstica del estudio microscópico de médula de $38.7 \%$, con una prevalencia de $32.2 \%$ para infección oportunista, la cual es mayor a la encontrada en nuestro estudio; sin embargo, cabe destacar que el doctor Madrigal y cols. realizaron su estudio en pacientes con estadio VIH-SIDA cuyo criterio de inclusión fue la presencia de pancitopenia febril y no febril, lo cual explicaría mejor aporte diagnóstico para determinar causas infecciosas (6).

Van Schalkwyk W y cols. (13) encontraron una prevalencia de infección oportunista con base en el estudio microscópico y microbiológico de médula ósea de $34.45 \%$, siendo el compromiso por micobacterias la entidad infecciosa más frecuente seguida por Criptococcus spp (13). Estos datos contrastan con los resultados del presente estudio, donde el compromiso por Histoplasma capsulatum fue la entidad oportunista más prevalente, representando la mayor parte de los casos informados como positivos tanto en el estudio microscópico (aspirado y/o biopsia) y microbiologico de médula ósea, seguida de compromiso por micobacterias e infeccion por Criptococcus spp. Así mismo difiere de lo reportado por otros estudios, donde muestran el compromiso por micobacterias como la infección más prevalente en el estudio de médula ósea $(7,15,18,19)$.

Varios estudios han evaluado posibles predictores clínicos y paraclínicos asociados al resultado positivo del estudio de médula ósea en el paciente con VIH $(2,4,5,13,18)$.Van Schalkwyk W y cols. (13) encontraron que un recuento de neutrófilos $<0.5 \times 10^{9} / \mathrm{L}$, nivel de hemoglobina $<6$ g/dL y un diagnóstico previo de tuberculosis fueron las condiciones asociadas con un diagnóstico nuevo en el estudio de médula ósea (13). En nuestro estudio, 93.4\% (86) tenían datos de la variable LDH, de éstos, sólo 8 (9.3\%) tenían un valor normal. Esto se correlaciona con los datos reportados por Santos E. y cols. (20), en cuya población estudiada la variable lactato deshidrogenasa fue normal en sólo $9.7 \%$. El presente estudio documentó que la variable LDH mayor o igual a $600 \mathrm{UI} / \mathrm{dL}$, aumenta la probabilidad en 5.4 veces de obtener un resultado positivo para infección oportunista en el estudio microsocópico de médula ósea. Sin embargo, reconocemos que el intervalo de confianza es amplio, lo que implica evaluar dicha asociación con un mayor tamaño de muestra para estimar mejor la relación con esta variable de interés.

Adicionalmente se obtuvieron otros resultados que a pesar de no hacer parte de los objetivos del presente estudio son importantes y ameritan su descripción. Se encontraron otras variables independientes que se relacionaron estadísticamente con un resultado positivo para infección oportunista en el estudio microscópico de médula ósea. Estas variables son: recuento de leucocitos, presencia de neutropenia, nivel de AST y ALT. Algunos de estos hallazgos fueron reportados por otros autores, en particular por Fernández A. y cols. (8), quienes documentaron que la presencia de trombocitopenia $\left(<75 \times 10^{9} / 1\right)$ y un nivel aspartato aminotransferasa (AST $\geq$ $100 \mathrm{U} / \mathrm{l})$ fueron factores asociados a una alta probabilidad de obtener un diagnóstico en el estudio de médula ósea en pacientes con VIH-SIDA con fiebre prolongada $(3,8)$. Aunque no fueron parte de los objetivos, estos hallazgos requieren confirmación en estudios posteriores diseñados para tal fin.

Santos E. y cols. (20) reportaron una sensibilidad y especificidad de la biopsia de médula ósea para detección de algún diagnóstico de 22 y $100 \%$ respectivamente. Igualmente para detección de etiología infecciosa informaron una sensibilidad y especificidad de 35 y $91 \%$ (20). Si bien las cifras anteriormente expuestas, indican un bajo rendimiento del estudio microscópico, en la presente investigación se demuestra que en una cuarta parte de los casos estudiados, el estudio microscópico de médula ósea permitió un diagnóstico precoz de entidades oportunistas, las cuales usualmente toman varias semanas cuando éstos se basan en sólo cultivos.

El estudio histopatológico de médula ósea en nuestro estudio reportó presencia de granulomas en cuatro casos, correspondiente a $4.8 \%$ del total de biopsias realizadas, que difiere de las cifras reportadas por otros investigadores $(5,13)$. El bajo porcentaje de identificación de granulomas en la muestra evaluada podría estar asociado en su mayor parte a un mayor grado de inmunosupresión en esta población, en la que no sería posible evidenciar los granulomas que requieren un mejor estado inmunológico para su formación.

Se han descrito algunos cambios morfológicos en la médula ósea de los pacientes con VIH-SIDA los cuales varían de acuerdo con los estadios de esta condición. En las 
fases iniciales de la infección se han descrito médulas óseas hipercelulares y en fases avanzadas hipocelularidad con displasia trilinaje (21). Como lo reportado por otros estudios, la plasmocitosis reactiva fue un hallazgo morfológico frecuente en los aspirados de médula ósea (22-24). A diferencia de lo informado por otros estudios, la mayoría de las biopsias analizadas fueron hipocelulares $(23,25,26)$. La displasia y la hiperplasia de la línea eritroide fueron los hallazgos más frecuentes luego de la plasmocitosis, alteraciones morfológicas que han sido extensamente descritas en la literatura en la médula ósea de los pacientes con VIH (21-26).

\section{Conclusiones}

Los resultados de este trabajo muestran que la frecuencia de identificación de infecciones oportunistas en pacientes con VIH-SIDA con base en el estudio microscópico de médula ósea muestra concordancia con lo que está publicado en la literatura. Para nuestro medio es una herramienta útil que contribuye a acelerar el proceso diagnóstico en una cuarta parte de los casos, lo cual puede repercutir en disminuir la morbilidad, costos y mortalidad asociada a oportunistas. Se requieren estudios prospectivos que contribuyan a aclarar las variables que se asocian a positividad del estudio con el propósito de optimizar la utilización de esta herramienta en áreas con epidemiología similar a la de Colombia y en poblaciones representativas de todo el espectro inmunológico con el que se pueden presentar los pacientes con VIH.

\section{Declaración de fuentes de financiación y posibles Conflictos de Interés}

Para la realización del presente estudio los autores manifiestan no haber recibido respaldo financiero alguno y declaran no presentar ningún conflicto de interés.

\section{Referencias}

1. Northfelt DW, Mayer A, Kaplan LD, Abrams DI, Hadley WK, Yajko DM, et al. The usefulness of diagnostic bone marrow examination in patients with human immunodeficiency virus (HIV) infection. J Acquir Immune Defic Syndr 1991; 4: 659-66.

2. Brook MG, Ayles H, Harrison C, Rowntree C, Miller RF. Diagnostic utility of bone marrow sampling in HIV positive patients. Genitourin Med. 1997;73: 117-21.

3. Gluckman RJ, Rosner F, Guarneri JJ. The diagnostic utility of bone marrow aspiration and biopsy in patients with acquired immunodeficiency syndrome. $J$ Natl Med Assoc. 1989; 81: 119-25.

4. Tanaka PY, Hadad DJ, Barletti SC, de Souza SA, Calore EE. Bone marrow biopsy in the diagnoses of infectious and non-infectious causes in patients with advanced HIV infection. J Infect. 2007; 54: 362-6.

5. Ciaudo M, Doco-Lecompte T, Guettier C, d'Agay MF, David F, Rioual N, et al. Revisited indications for bone marrow examinations in HIV-infected patients. Eur J Haematol. 1994; 53: 168-74.

6. Madrigal H, Hernández G. Utilidad del estudio microscopico de médula ósea en pacientes con infeccion por VIH y pancitopenia. Gac Med Mex.2006; 142: 13-7.

7. Luther JM, Lakey DL, Larson RS, Kallianpur AR, D'Agata E, Cousar JB, et al. Utility of bone marrow biopsy for rapid diagnosis of febrile illnesses in patients with human immunodeficiency virus infection. South Med J. 2000; 93: 692-7.

8. Fernandez-Aviles F, Ribera JM, Romeu J, Batlle M, Navarro JT, Manterola JM, et al. [The usefulness of the bone marrow examination in the etiological diagnosis of prolonged fever in patients with HIV infection]. Med Clin (Barc) 1999; 112: 641-5.

9. Morais JC, Machado M, Biasoli I, Barroso PF, Milito C, Spector N. Changing patterns of AIDS: impact on the indications and diagnostic yield of bone marrow biopsies. Braz J Infect Dis. 2010; 14: 419-21.

10. Corcoran GR, Al-Abdely H, Flanders CD, Geimer J, Patterson TF. Markedly elevated serum lactate dehydrogenase levels are a clue to the diagnosis of disseminated histoplasmosis in patients with AIDS. Clin Infect Dis. 1997; 24: 942-4.

11. Butt AA, Michaels S, Kissinger $P$. The association of serum lactate dehydrogenase level with selected opportunistic infections and HIV progression.Int J Infect Dis. 2002; 6: 178-81.

12. Butt AA, Michaels S, Greer D, Clark R, Kissinger P, Martin DH. Serum LDH level as a clue to the diagnosis of histoplasmosis. AIDS Read 2002; 12: 317-21.

13. Van Schalkwyk WA, Opie J, Novitzky N. The diagnostic utility of bone marrow biopsies performed for the investigation of fever and/or cytopenias in HIV-infected adults at Groote Schuur Hospital, Western Cape, South Africa. Int J Lab Hematol. 2011; 33: 258-66.

14. Miralles P, Moreno S, Perez-Tascon M, Cosin J, Diaz MD, Bouza E. Fever of uncertain origin in patients infected with the human immunodeficiency virus. Clin Infect Dis. 1995; 20:872-5.

15. Pande A, Bhattacharyya M, Pain S, Ghosh A, Samanta A. Diagnostic yield of bone marrow examination in HIV associated FUO in ART naive patients. J Infect Public Health. 2010; 3: 124-9.

16. Bissuel F, Leport C, Perronne C, Longuet P, Vilde JL. Fever of unknown origin in HIV-infected patients: a critical analysis of a retrospective series of 57 cases. J Intern Med. 1994; 236: 529-35.

17. Engels E, Marks PW, Kazanjian P. Usefulness of bone marrow examination in the evaluation of unexplained fevers in patients infected with human immunodeficiency virus. Clin Infect Dis. 1995; 21: 427-8.

18. Karstaedt AS, Pantanowitz L, Omar T, Sonnendecker HE, Patel M. The utility of bone-marrow examination in HIV-infected adults in South Africa.QJM 2001; 94:101-5.

19. Mayo J, Collazos J, Martinez E. Fever of unknown origin in the HIV-infected patient: new scenario for an old problem. Scand J Infect Dis. 1997; 29: 327-36.

20. Santos ES, Raez LE, Eckardt P, DeCesare T, Whitcomb CC, Byrne GE, Jr. The utility of a bone marrow biopsy in diagnosing the source of fever of unknown origin in patients with AIDS. J Acquir Immune Defic Syndr. 2004; 37: 1599-603.

21. Bain BJ. The haematological features of HIV infection. Br J Haematol. 1997; 99: $1-8$

22. Castella A, Croxson TS, Mildvan D, Witt DH, Zalusky R. The bone marrow in AIDS. A histologic, hematologic, and microbiologic study. Am J Clin Pathol. 1985; 84: 425-32.

23. Shenoy CM, Lin JH. Bone marrow findings in acquired immunodeficiency syndrome (AIDS). Am J Med Sci. 1986; 292: 372-5.

24. Zhao X, Sun NC, Witt MD, Keller M, Niihara Y. Changing pattern of AIDS a bone marrow study. Am J Clin Pathol. 2004; 121: 393-401.

25.Zon LI, Arkin C, Groopman JE. Hematologic manifestations of the human immune deficiency virus (HIV). Br J Hematol. 1987; 66: 251-6.

26. Frontiera M, Myers AM. Peripheral blood and bone marrow abnormalities in the acquired immunodeficiency syndrome. West J Med. 1987; 147: 157-60. 